\title{
Traffic characteristics and queueing behavior of discrete-time on-off sources
}

\author{
K. Laevens and H. Bruneel ${ }^{1}$ \\ SMACS Research Group, University of Ghent \\ Sint-Pietersnieuwstraat 41, B-9000 Ghent, Belgium \\ Email: kl,hb@lci.rug.ac.be
}

\begin{abstract}
In this paper, some results are presented from an attempt to study - in a discrete-time setting - the phenomenon of long-range dependence. For a well-known source model, traffic characteristics such as the power spectral density and the index of dispersion for counts are analyzed. Based on these characteristics, the distinction between short-range and long-range dependence is touched upon. The traffic generated by a superposition of sources is also studied, whereby the case of an infinite number of sources gets special attention. In this, the discrete-time version of the $M / G / \infty$ queue model is called upon. Finally, some results pertaining to the queueing behavior of such traffic are discussed.
\end{abstract}

\section{Introduction}

Since the notions 'long-range dependence' and 'self-similarity' $[1,10$, 16] have been brought to the attention of the teletraffic community, a tremendous amount of research effort has been spent on the subject. Issues of importance hereby are traffic analysis and modelling, and queueing analysis. Fluid-flow models $[2,8,9,12]$ and the related fractional Brownian storage $[13,14,18]$, combined with elements of largedeviations theory, seem to have been the most successful modelling approaches yet, be it sometimes at the cost of complex mathematics.

\footnotetext{
${ }^{1}$ The authors wish to thank the Belgian Federal Office for Scientific, Technical and Cultural Affairs (DWTC) and the Flemish Fund for Scientific Research (FWOVlaanderen) for support of this research.
} 
What we present here, are some results from an attempt to study these notions in a discrete-time setting. As a source model, we opted for the well-known on-off source. Long-range dependence is expected to emerge when heavy-tailed distributions for e.g. the durations of the onperiods come into play. These heavy-tailed distributions typically lead to probability generating functions - one of the basic tools of our analysis having a branch point at $z=1$. This branch point affects the use that is made of residue theory and urges us to reconsider some results obtained for 'light-tailed' distributions. This work is part of ongoing research and additional study is required to fill in the remaining gaps and to provide a more solid mathematical framework.

The paper is structured as follows. In the next section, the on-off source model is introduced. In Section 3, traffic characteristics such as the autocovariance function and the index of dispersion for counts are analyzed. The difference between short- and long-range dependent sources is examined in Section 4. In Section 5, the superposition of $N$ sources is considered, whereby the case $N \rightarrow \infty$ receives the most attention. It leads to a discrete-time $M / G / \infty$ queue model, recently also studied in e.g. $[15,19]$. In Section 6 , two possible approaches to the analysis of the queueing behavior - a Benes approach and a slot-to-slot approach - are discussed. Conclusions are drawn in Section 7.

\section{The source model}

An on-off source alternates between two states : the on-state - wherein one cell is generated per slot - and the off-state - wherein no cells are generated, as shown in Figure 1.

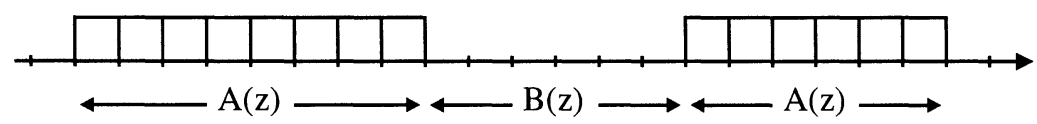

Figure 1: A discrete-time on-off source.

The durations, expressed in numbers of slots, of the visits to the on-state - called the on-periods - are iid random variables (rv's) characterized by the probability density function (pdf) $a(n)=\operatorname{Pr}\left[\tau_{A}=n\right]$ 
$(n=1,2, \ldots)$ or the associated probability generating function (pgf)

$$
A(z)=E\left[z^{\tau_{A}}\right]=\sum_{n=1}^{+\infty} a(n) z^{n}
$$

Likewise, the durations of the off-periods are iid rv's characterized by the pdf $b(n)=\operatorname{Pr}\left[\tau_{B}=n\right]$ or the pgf $B(z)=E\left[z^{\tau_{B}}\right]$. Hereby, $\tau_{A}$ and $\tau_{B}$ were used to denote the duration of a generic on- or off-period respectively. Durations of on- and off-periods are mutually independent, their mean values equal

$$
E\left[\tau_{A}\right]=A^{\prime}(1) \quad \text { and } \quad E\left[\tau_{B}\right]=B^{\prime}(1)
$$

respectively. Unless otherwise stated, these values are assumed to be finite. As a consequence, the stationary version of the process, which is of interest here, exists. Variances are given by

$$
\sigma_{A}^{2}=\operatorname{Var}\left[\tau_{A}\right]=A^{\prime \prime}(1)+A^{\prime}(1)-A^{\prime}(1)^{2}
$$

and

$$
\sigma_{B}^{2}=\operatorname{Var}\left[\tau_{B}\right]=B^{\prime \prime}(1)+B^{\prime}(1)-B^{\prime}(1)^{2}
$$

and can be either finite or infinite.

Two distributions that are frequently encountered in the rest of the analysis, are the distributions of the remaining durations $\tau_{A}^{*}$ or $\tau_{B}^{*}$ of the on- or off-period to which a randomly chosen slot belongs (not counting the arbitrary slot itself). It can be shown, see e.g. [3], that, for onperiods, this distribution is given by

$$
a^{*}(n)=\operatorname{Pr}\left[\tau_{A}^{*}=n\right]=\operatorname{Pr}\left[\tau_{A}>n\right] / E\left[\tau_{A}\right] \quad n=0,1, \ldots
$$

and mutatis mutandis for the off-periods. The associated pgf's take the form

$$
A^{*}(z)=\frac{A(z)-1}{A^{\prime}(1)(z-1)} \quad \text { and } \quad B^{*}(z)=\frac{B(z)-1}{B^{\prime}(1)(z-1)}
$$

The number of cells generated by the source during slot $k$, either 0 or 1 , will be denoted by $q_{k}$. The average of $q_{k}$ can be expressed as

$$
\lambda=E\left[q_{k}\right]=\frac{E\left[\tau_{A}\right]}{E\left[\tau_{A}\right]+E\left[\tau_{B}\right]}
$$


and its variance as

$$
\sigma^{2}=\operatorname{Var}\left[q_{k}\right]=\lambda(1-\lambda)
$$

In the next section, some further characteristics of the traffic process $q_{k}$ are derived.

\section{$3 \quad$ Traffic characteristics}

\subsection{The power spectral density}

The Fourier-transform

$$
S(f)=\sum_{m=-\infty}^{+\infty} C(m) e^{j 2 \pi m f}
$$

of the autocovariance function

$$
C(m)=E\left[\left(q_{0}-\lambda\right)\left(q_{m}-\lambda\right)\right]
$$

is known as the power spectral density of the traffic process. Studies such as [5] or [11] come to the conclusion that the power spectral density at the low frequencies has a serious impact on the queueing behavior of the traffic. The power spectral density of long-range dependent traffic behaves totally different at these frequencies than that of short-range dependent traffic. We return on this in Section 4.

In Appendix A, it is shown that

$$
S(f)=\sigma^{2}\left(1+Q\left(e^{j 2 \pi f}\right)+Q\left(e^{-j 2 \pi f}\right)\right)
$$

whereby

$$
\sum_{m=1}^{+\infty} C(m) z^{m}=\sigma^{2} Q(z)=\sigma^{2} z \frac{P(z)-1}{z-1}
$$

with

$$
P(z)=\frac{A(z)-1}{A^{\prime}(1)(z-1)} \cdot \frac{B(z)-1}{B^{\prime}(1)(z-1)} \cdot \frac{\left[A^{\prime}(1)+B^{\prime}(1)\right](z-1)}{A(z) B(z)-1}
$$


Given $A(z)$ and $B(z), S(f)$ is easily evaluated. As such, $C(m)$ can be calculated by numerical transform inversion, as outlined in e.g. [4]. Further, it follows from residue theory that

$$
C(m)=\operatorname{Res}\left[\sigma^{2} \frac{Q(z)}{z^{m+1}}\right]_{z=0}
$$

If the only singularities of $Q(z)$ are poles outside the unit disk, say $z_{i}$, the above can be rewritten as

$$
C(m)=-\sum_{i} \operatorname{Res}\left[\sigma^{2} \frac{Q(z)}{z^{m+1}}\right]_{z=z_{i}}
$$

For $m$ large, one can then retain only the dominant contribution and obtain an approximation for $C(m)$. In that case, the decay of the autocovariance will be dominated by a geometric term, i.e., the process will be short-range dependent, as to be discussed later on. For long-range dependent processes, (one of) the generating functions $A(z)$ or $B(z)$ will have a branch point at $z=1$, due to the 'heavy tail' of the distribution involved. Then, the result from residue theory should be reformulated and a non-geometric term will dominate the decay of the autocovariance function.

A quantity of interest in Section 4 is the so-called 'DC-component' of the power spectral density, given by

$$
S(0)=\sigma^{2}\left\{\frac{\sigma_{A}^{2}}{E\left[\tau_{A}\right]}+\frac{\sigma_{B}^{2}}{E\left[\tau_{B}\right]}-\frac{\sigma_{A}^{2}+\sigma_{B}^{2}}{E\left[\tau_{A}\right]+E\left[\tau_{B}\right]}\right\}
$$

\subsection{Index of dispersion for counts}

Another well-known traffic characteristic, the index of dispersion for counts, was discussed in e.g. [6]. It is defined as

$$
\operatorname{IDC}(m)=\frac{\operatorname{Var}\left[q_{1}+\ldots+q_{m}\right]}{E\left[q_{1}+\ldots+q_{m}\right]}
$$

and is related to the autocovariance function $C(m)$ by [6]

$$
\operatorname{IDC}(m)=\frac{1}{\lambda} \sum_{k=-m}^{+m}\left(1-\frac{|k|}{m}\right) C(k)
$$

In this respect, both traffic characteristics convey the same information. 
Consider the double transform

$$
J(z, t)=\sum_{m=1}^{+\infty} t^{m} E\left[z^{q_{1}+\ldots+q_{m}}\right]
$$

of the number of arrivals during $m$ consecutive slots. One can show that, for the on-off source model used here,

$$
\begin{aligned}
J(z, t)=\frac{1}{A^{\prime}(1)+B^{\prime}(1)} & \frac{A^{\prime}(1) z t}{1-z t}+\frac{B^{\prime}(1) t}{1-t} \\
& \left.-\frac{A^{\prime}(1) B^{\prime}(1) t^{2}(z-1)^{2} A^{*}(z t) B^{*}(t)}{(1-z t)(1-t)(1-A(z t) B(t))}\right)
\end{aligned}
$$

as explained in Appendix B.

Taking the first-order partial derivative with respect to $z$ at $z=1$, one finds

$$
\left.\frac{\partial}{\partial z} J(z, t)\right|_{z=1}=\sum_{m=1}^{+\infty} t^{m} E\left[q_{1}+\ldots+q_{m}\right]=\frac{1}{A^{\prime}(1)+B^{\prime}(1)} \cdot \frac{A^{\prime}(1) t}{(1-t)^{2}}
$$

from which follows the obvious result

$$
E\left[q_{1}+\ldots+q_{m}\right]=m \lambda
$$

Taking the second-order partial derivative with respect to $z$ at $z=1$, one finds after some further manipulation

$$
\sum_{m=1}^{+\infty} t^{m} \operatorname{Var}\left[q_{1}+\ldots+q_{m}\right]=\sigma^{2} t \frac{1+t-2 t P(t)}{(1-t)^{3}}
$$

From this, one can derive the result

$$
\Phi(t)=\sum_{m=1}^{+\infty} t^{m} \operatorname{IDC}(m)=\frac{\sigma^{2}}{\lambda} \int_{0}^{t} \frac{1+s-2 s P(s)}{(1-s)^{3}} d s
$$

As for $C(m)$, transform inversion, be it numerical or based on residue theory, of equation (4) or (5), then yields $\operatorname{Var}\left[p_{1}+\ldots+p_{m}\right]$ or IDC $(m)$. (For this, equation (4) seems more appropriate, since it is of a simpler nature.) Of course, the same problems with transform inversion as for the power spectral density mentioned above will emerge when long-range dependent processes are considered. 
From (5), one can also derive that

$$
\operatorname{IDC}(+\infty)=\frac{\sigma^{2}}{\lambda}\left\{\frac{\sigma_{A}^{2}}{\tau_{A}}+\frac{\sigma_{B}^{2}}{\tau_{B}}-\frac{\sigma_{A}^{2}+\sigma_{B}^{2}}{\tau_{A}+\tau_{B}}\right\}=\frac{S(0)}{\lambda}
$$

as follows from equations (3) and (2), see [6].

\subsection{A special case}

A source whereby transitions from one state to the other form a twostate Markov chain, is a special case of the more general on-off source model considered here. It is obtained when the distributions for the durations of the on- and off-periods are geometric with mean $1 / \alpha$ and $1 / \beta$ respectively, i.e., when

$$
A(z)=\frac{\alpha z}{1-(1-\alpha) z} \quad \text { and } \quad B(z)=\frac{\beta z}{1-(1-\beta) z}
$$

The transition probabilities from the on- to the off-state and vice versa are then given by $\alpha$ and $\beta$ respectively. Traffic characteristics for this specific model can be calculated using standard techniques from Markov chain analysis - see e.g. [7]. One finds, amongst others,

$$
C(m)=\sigma^{2} \delta^{m}
$$

and

$$
\operatorname{Var}\left[q_{1}+\ldots+q_{m}\right]=\sigma^{2}\left(m+\frac{2 \delta}{1-\delta}\left(m-\frac{1-\delta^{m}}{1-\delta}\right)\right)
$$

Hereby $\delta=1-\alpha-\beta$ is one of the two eigenvalues of the transition matrix governing the Markov chain, the other being 1 . It has been verified that these results are in full agreement with the ones obtained in the previous subsections for the more general model. For instance, $1 / \delta$ is the only pole of $P(t)$, which is now given by

$$
P(t)=\frac{1-\delta}{1-\delta t}
$$

Transform inversion is then quite straightforward and leads to the above expressions. 


\section{Short-range versus long-range dependence}

By definition [10], long-range dependence is present when

$$
\sum_{m=-\infty}^{+\infty} C(m)=S(0)=+\infty
$$

Recalling equation (2), we see that this will be the case when, for instance, $\sigma_{A}^{2}=\operatorname{Var}\left[\tau_{A}\right]$ is infinite (given $E\left[\tau_{A}\right]$ is finite), the so-called 'infinite variance syndrome' [10]. This condition implies that $A(z)$ has a singularity at $z=1$, that cannot be a pole since $A(1)=1$, but is a branch point. As a consequence, the distribution $a(n)$ of which $A(z)$ is the pgf, will not decay geometrically, but hyperbolically, i.e., $a(n)$ will have a heavy tail.

Well-known heavy-tailed distributions of a continuous random variable are e.g. the Pareto-distribution [16]. In search of a versatile heavytailed distribution for a discrete-time random variable, we focussed on a distribution based on the hypergeometric function

$$
F(\alpha, \beta ; \gamma ; z)=\sum_{n=0}^{+\infty} \frac{\Gamma(\alpha+n) \Gamma(\beta+n) \Gamma(\gamma)}{\Gamma(\alpha) \Gamma(\beta) \Gamma(\gamma+n) n !} z^{n}
$$

To be more specific, we used a generating function of the form

$$
A(\alpha, \beta ; \gamma ; z)=z \frac{F(\alpha, \beta ; \gamma ; z)}{F(\alpha, \beta ; \gamma ; 1)}
$$

The resulting distribution seems versatile in the sense that its pgf is based on a well-studied function for which numerical procedures are available [17] and that it has three (real-valued) parameters, which can be fitted to yield e.g. a given mean and tail decay. The pgf has a branch point at $z=1$ and the tail of the distribution decays hyperbolically as

$$
a(\alpha, \beta ; \gamma ; n) \approx \frac{\Gamma(\gamma-\alpha) \Gamma(\gamma-\beta)}{\Gamma(\alpha) \Gamma(\beta) \Gamma(\gamma-\alpha-\beta)} n^{-(\gamma-\alpha-\beta+1)}
$$

for $n \gg 1$. The variance is infinite and long-range dependence will result whenever $1<\gamma-\alpha-\beta \leq 2$. (The lower bound is required for the mean to be finite.)

Throughout this paper, three different distributions for the on-periods will be used for illustrative purposes, as summarized in Table 1: a lighttailed geometric distribution (A), a heavy-tailed distribution (B) of the 
form (6) with infinite variance and a third distribution (C), also of the form (6), with finite variance but infinite third moment. Including the latter distribution will allow us to distinguish between 'long-range dependent' features and features originating from a 'heavy tail'. All three distributions have mean $E\left[\tau_{A}\right]=100.0$ slots.

\begin{tabular}{|c|c|c|}
\hline & variance & tail behavior \\
\hline $\mathrm{A}$ & $<+\infty$ & $\sim z_{0}^{-n}$ \\
\hline $\mathrm{B}$ & $=+\infty$ & $\sim n^{-2.5}$ \\
\hline $\mathrm{C}$ & $<+\infty$ & $\sim n^{-3.5}$ \\
\hline
\end{tabular}

Table 1: Three different distributions

In Figure 2, where these distributions were plotted, the slow decay of the tails of distributions (B) and (C) clearly shows. In Figure 3, a $\log$-log plot of the complementary cummulative distribution, this is even more apparent.

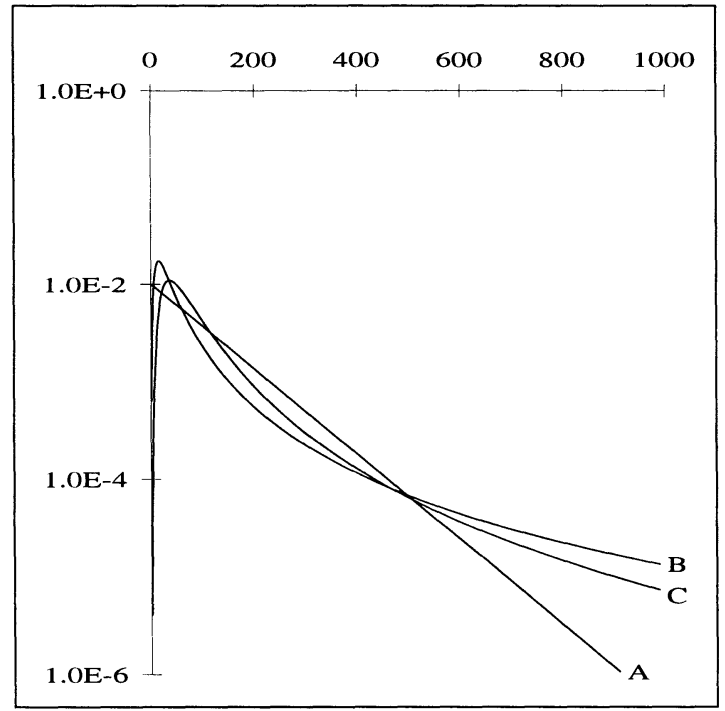

Figure 2: Tail behavior, $\log \operatorname{Pr}\left[\tau_{A}=n\right]$ versus $n$, for various types of distributions.

Power spectral densities of sources with on-periods as introduced above, are shown in Figure 4. A geometrically distributed off-period, with mean 25.0 slots, was assumed for all cases, yielding a traffic intensity of 0.8 Erlang. It is known [10] that, for long-range dependent 


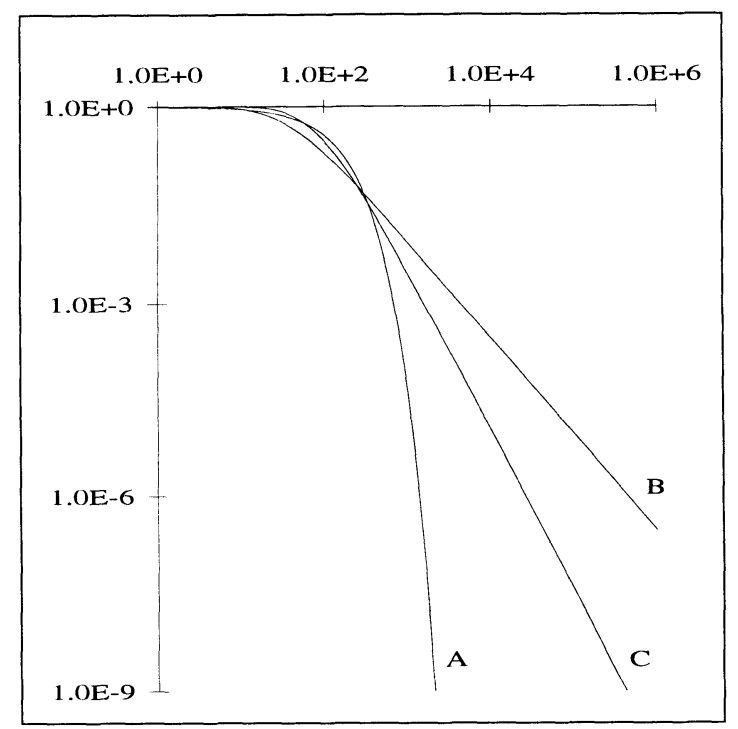

Figure 3: Tail behavior, $\log \operatorname{Pr}\left[\tau_{A}>n\right]$ versus $\log n$, for various types of distributions.

sources, $S(f) \sim f^{-\nu}$ or $\log S(f) \sim-\nu \log f$, when $f \rightarrow 0$, while for short-range dependent sources, $\log S(f) \sim \log S(0)$. Both types of behavior are clearly distinguishable in Figure 4 . Note that while the tail of distribution (C) is also hyperbolic and thus 'heavy', it decays too fast to yield long-range dependence in the strict sense. Corresponding autocovariance functions, obtained numerically, are shown in Figure 5.

Evidence for long-range dependence is also present in the sample traces presented in Figure 6 . The figure was obtained by aggregating the traffic over various timescales $\left(1,10,100, \ldots 10^{6}\right.$ slots respectively). The traffic was generated by a superposition (see Section 5 ) of 5 iid sources and the total traffic intensity is 0.8 Erlang. In traffic of type (B), large fluctuations occur over large time-scales, while for traffic of type (A) and (C), fluctuations quickly die out as the time scale increases.

\section{Superposition}

\section{$5.1 \quad \mathrm{~N}$ sources}

Traffic characteristics of a superposition of $N$ identical and independent sources, are easily derived from those of a single source. Assume the $N$ sources generate the aggregate traffic stream $p_{k}$. The mean total arrival 


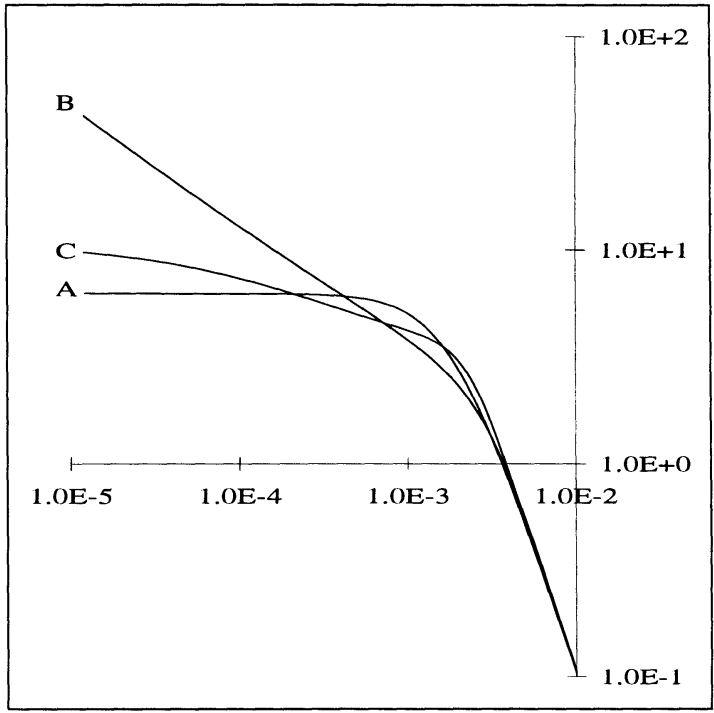

Figure 4: Power spectral density, $\log S(f)$ versus $\log f$, for various types of source.

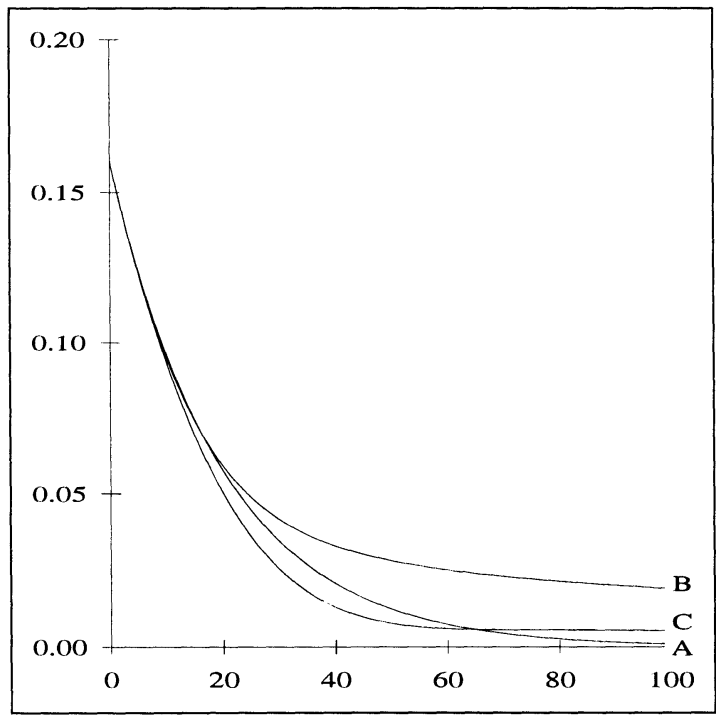

Figure 5: Autocovariance function, $C(m)$ versus $m$, for various types of sources.

rate is then $\lambda_{T}=E\left[p_{k}\right]=N \lambda$. Other traffic characteristics are given by 

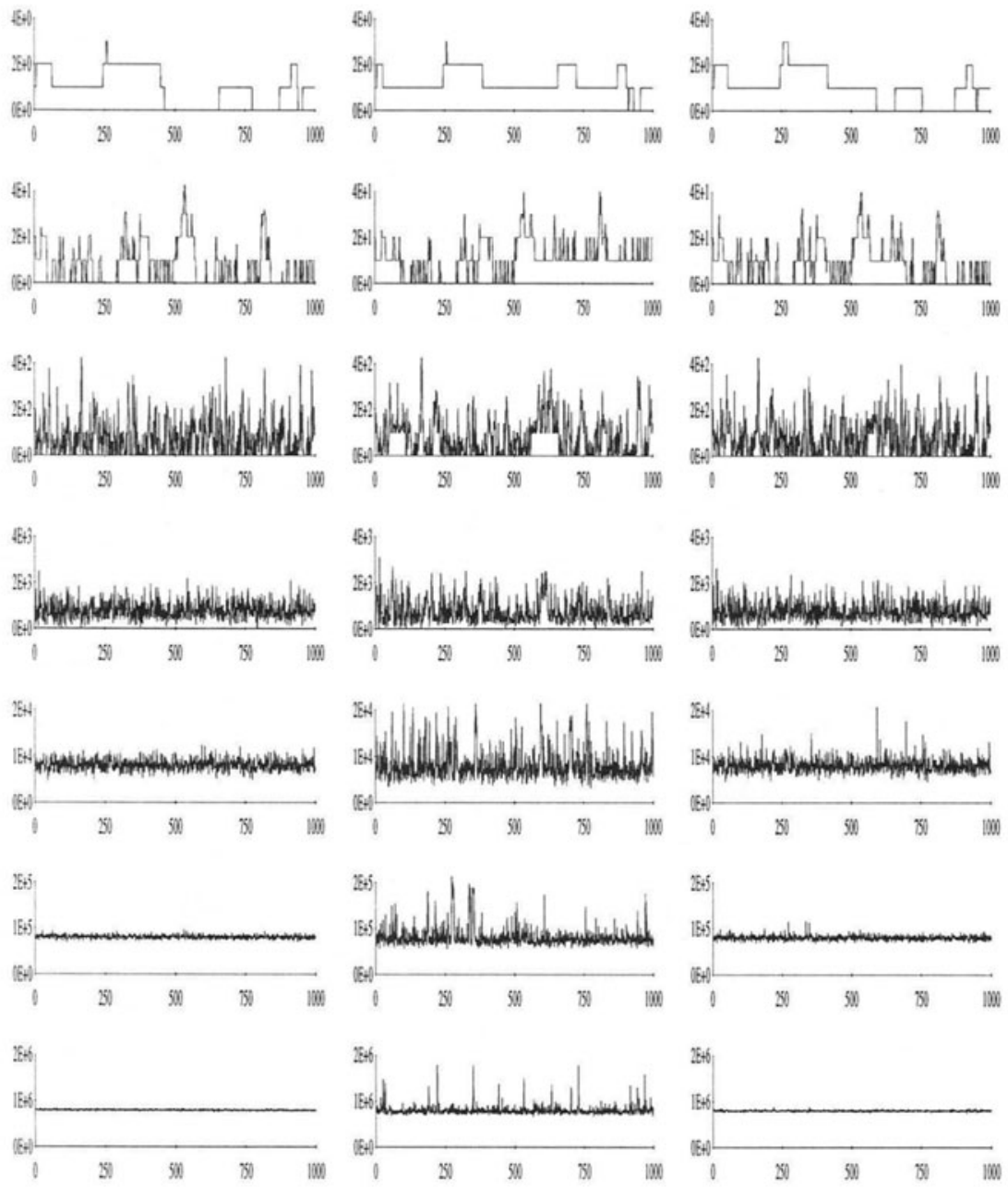

Figure 6: Aggregated traffic traces for source types A, B and C (left to right).

e.g.

$$
S_{(N)}(f)=N S(f)
$$


and

$$
E\left[z^{p_{1}+\ldots+p_{m}}\right]=\left(E\left[z^{q_{1}+\ldots+q_{m}}\right]\right)^{N}
$$

From the latter follows easily

$$
\operatorname{Var}\left[p_{1}+\ldots+p_{m}\right]=N \operatorname{Var}\left[q_{1}+\ldots+q_{m}\right]
$$

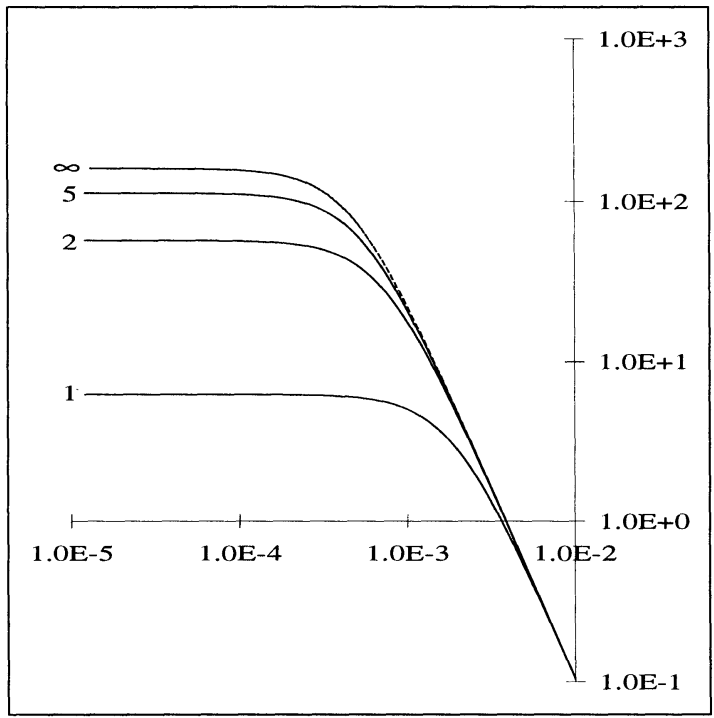

Figure 7: Power spectral density, $\log S(f)$ versus $\log f$, for a superposition of sources of type A.

For illustrative purposes, Figures 7 and 8 show the power spectral density for a superposition of $1,2,5$ and an infinite number of sources. (The latter case is treated in more detail below.) Figure 7 is for a shortrange dependent source of type (A), Figure 8 for a long-range dependent source of type (B). The total arrival rate was kept constant at 0.8 Erlang by varying the mean duration of the off-periods.

\section{$5.2 N \rightarrow+\infty$}

An interesting case is that whereby the number of superpositioned sources grows infinitely, with given $\lambda_{T}$ and $A(z)$. As illustrated by Figures 7 and 8 , traffic characteristics quickly approach their limiting values as 


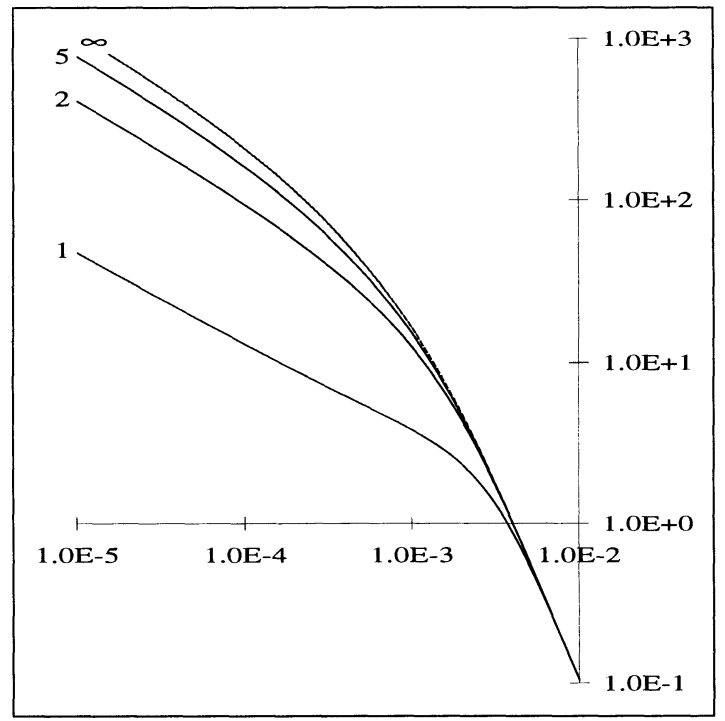

Figure 8: Power spectral density, $\log S(f)$ versus $\log f$, for a superposition of sources of type B.

the number of sources increases. For the power spectral density, we find by taking a limit

$$
S_{(\infty)}(f)=\lim _{N \rightarrow+\infty} S_{(N)}(f)=\lambda_{T}\left(1+Q_{(\infty)}\left(e^{j 2 \pi f}\right)+Q_{(\infty)}\left(e^{-j 2 \pi f}\right)\right)
$$

whereby

$$
Q_{(\infty)}(z)=z \frac{A^{*}(z)-1}{z-1}
$$

Concerning the total number of arrivals in $m$ consecutive slot, see Section 3.2 , derivation of

$$
J_{(\infty)}(z, t)=\lim _{N \rightarrow \infty} \sum_{m=1}^{+\infty} t^{m} E\left[z^{p_{1}+\ldots+p_{m}}\right]
$$

through a limiting procedure seems more cumbersome. For e.g. the variance of that number, on the contrary, we easily obtain

$$
\lim _{N \rightarrow \infty} \sum_{m=1}^{+\infty} t^{m} \operatorname{Var}\left[p_{1}+\ldots+p_{m}\right]=\lambda_{T} \frac{1+t-2 t A^{*}(t)}{(1-t)^{3}} t
$$


through equations (4) and (7). Numerical or approximate transform inversion, then again yields $C(m)$ or $\operatorname{Var}\left[p_{1}+\ldots+p_{m}\right]$.

By observing, however, that the number of arrivals in a slot is equivalent with the number of customers in a discrete-time $G I-G-\infty$ queue, the equivalent of the continuous time $M / G / \infty$ queue, some more results can be obtained. Recently this model has been studied in e.g. [15, 19].

One can show that the numbers of newly arriving 'customers' in each slot, become iid rv's with a Poisson distribution, with mean $\lambda^{*}=$ $\lambda_{T} / A^{\prime}(1)$ and pgf

$$
\exp \left\{\lambda^{*}(z-1)\right\}
$$

The service times of the customers are, of course, also iid rv's with pgf $A(z)$, the on-time distribution. By analyzing this equivalent queue model on a slot-to-slot basis, it is possible to derive e.g. that

$$
C(m)=\lambda^{*} \sum_{k=m}^{+\infty} \operatorname{Pr}\left[\tau_{A}>k\right]
$$

Note that this is in full agreement with equation (8) derived above. This expression illustrates once more that light-tailed on-periods lead to short-range dependence, since

$$
\operatorname{Pr}\left[\tau_{A}=m\right] \sim z_{0}^{-m} \Rightarrow C(m) \sim z_{0}^{-m} \Rightarrow \sum_{m=-\infty}^{+\infty} C(m)<+\infty
$$

On the other hand, for heavy-tailed on-periods one has

$$
\operatorname{Pr}\left[\tau_{A}=m\right] \sim m^{-q} \Rightarrow C(m) \sim m^{-(q-2)} \Rightarrow \sum_{m=-\infty}^{+\infty} C(m)=+\infty
$$

when $2<q \leq 3$. Infinite variances for the on-periods thus lead to longrange dependence. Note, however, that also for $q>3$, as for traffic of type (C), the autocovariance function may decay quite slowly, i.e., correlation may extend over long time periods. It does not, however, lead to long-range dependence in the strict sense.

One can further derive that

$$
\begin{aligned}
& E\left[z^{p_{1}+\ldots+p_{m}}\right]=\exp \left\{\lambda^{*} \sum_{k=m}^{+\infty} \operatorname{Pr}\left[\tau_{A}>k\right]\left(z^{m}-1\right)\right. \\
& \left.+\lambda^{*} \sum_{k=0}^{m-1} \operatorname{Pr}\left[\tau_{A}>k\right]\left(z^{k}-1\right)+\lambda^{*} \sum_{k=0}^{m-1} \operatorname{Pr}\left[\tau_{A}>k\right](m-k) z^{k}(z-1)\right\}
\end{aligned}
$$


The first two sums in the RHS represent the contribution of 'old' sources, i.e., source that were already active prior to slot 1 . The last sum represents the contribution of sources that started generating cells during slot 1 or later. Taking derivatives and performing some algebra, one obtains

$$
\operatorname{Var}\left[p_{1}+\ldots+p_{m}\right]=m^{2} \lambda_{T}-\lambda^{*} \sum_{k=0}^{m-1} \operatorname{Pr}\left[\tau_{A}>k\right](m-k)(m-k-1)
$$

in agreement with the result obtained through a limiting procedure.

For $m=1$, the above pgf reduces to

$$
E\left[z^{p_{1}}\right]=\exp \left\{\lambda^{*}(z-1) A^{\prime}(1)\right\}=\exp \left\{\lambda_{T}(z-1)\right\}
$$

The distribution of the number of active sources or, equivalently, the total number of cells generated in a random slot, is thus Poisson and function of the load $\lambda_{T}$ only. This 'marginal' distribution is a rather smooth distribution and is in no way influenced by the exact form of the distribution of the on-periods. The latter does, however, strongly affect the correlation structure of process.

An interesting property of the $G I-G-\infty$ arrival processes is that the aggregation of two or more such processes is again of that type. This is a consequence of the fact that the arrival process of new 'customers' is Poisson. The 'parameters' of the aggregated $G I-G-\infty$ arrival process are given by

$$
\lambda^{*}=\lambda_{1}^{*}+\cdots+\lambda_{N}^{*}
$$

and

$$
A(z)=\frac{\lambda_{1}^{*} A_{1}(z)+\cdots+\lambda_{N}^{*} A_{N}(z)}{\lambda_{1}^{*}+\cdots+\lambda_{N}^{*}}
$$

From this, it is easily seen that the tail of the aggregated message length will be dominated by the heaviest tale of the constituent message lengths. In other words, long-range dependent processes will dominate over shortrange dependent ones.

\section{$6 \quad$ Queueing}

Two approaches seem promising to analyze the queueing behavior of traffic of the type described above when it is fed into a single-server 
system. The first is based on the Benes formula, the second on a slotto-slot approach. At the time, however, it is by no means clear whether these approaches will lead to 'practical' results, such as an approximate formula for the tail of the distribution of the system contents.

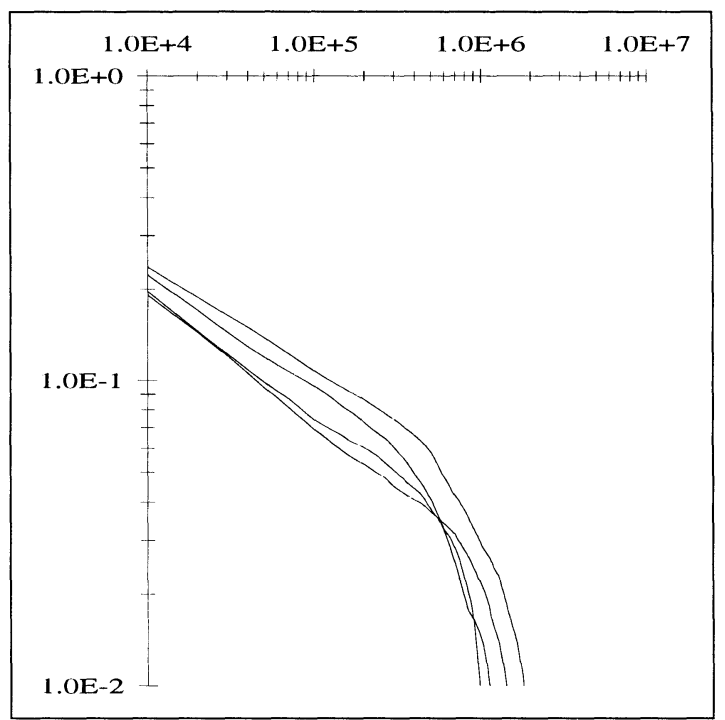

Figure 9: $\log \operatorname{Pr}[u>n]$ versus $\log n$, simulations for $G I-G-\infty$ traffic of type B.

Simulation results, shown in Figures 9 and 10, for a $G I-G-\infty$ arrival process of type $\mathrm{B}$ and $\mathrm{C}$ respectively with intensity 0.8 Erlang, give an indication of the magnitude of the queues - denoted by the variable $u$ that can build up. For instance, from Figure 9, we learn that for the longrange dependent case, the queue exceeds the order of $10^{5}$ cells during $10 \%$ of the time. For the other case, the magnitude of the queue is about a hundred times smaller, but still very large. Although the simulations are too crude to draw detailed conclusions, the figures already point towards a hyperbolic decay of the queue contents (a straight line in a $\log -\log$ plot).

\subsection{The Benes approach}

The system contents - observed at the beginning of a slot and denoted $u_{k}$ for slot $k$ - is governed by the equation

$$
u_{k+1}=p_{k}+\left[u_{k}-1\right]^{+}=p_{k}+\max \left(u_{k}-1,0\right)
$$




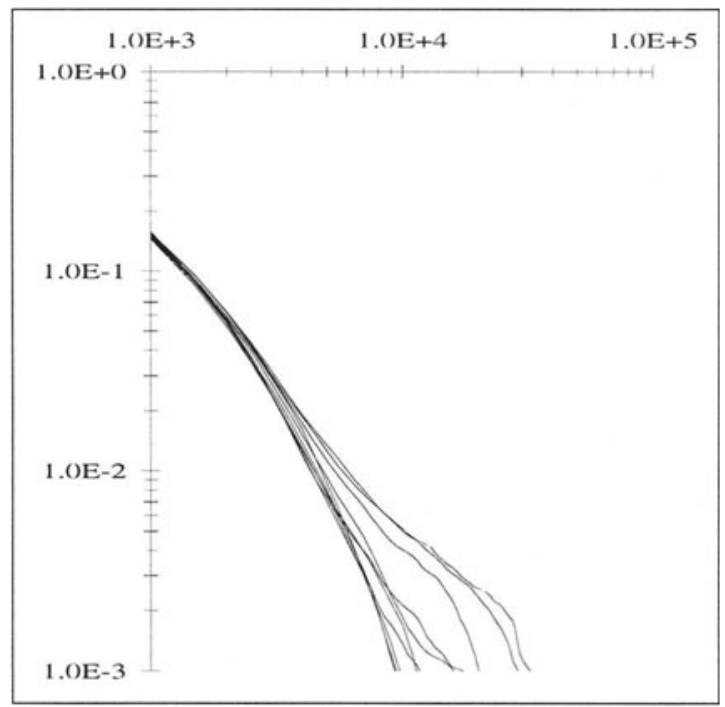

Figure 10: $\log \operatorname{Pr}[u>n]$ versus $\log n$, simulations for $G I-G-\infty$ traffic of type C.

The 'Benes result' $[12,18]$ for this system reads

$$
u_{k+1}=\max _{l \geq 0}\left(p_{k}+p_{k-1}+\ldots+p_{k-l}-l\right)
$$

from which one obtains

$$
\begin{aligned}
& \operatorname{Pr}\left[u_{k+1}>m\right]= \\
& \quad \sum_{l=0}^{+\infty} \operatorname{Pr}\left[p_{k}+p_{k-1}+\ldots+p_{k-l}>m+l \mid u_{k-l}=0\right] \operatorname{Pr}\left[u_{k-l}=0\right]
\end{aligned}
$$

This result is appealing, since it provides a formula for $\operatorname{Pr}\left[u_{k+1}>m\right]$ irrespective of the precise nature of the arrival process. Also, a similar expression can be derived for systems with service capacity larger than 1 or variable service capacity. Intriguing questions are what the link is between this general result and the general observation made in e.g. $[5,11]$ concerning the impact of the power spectral density at low frequencies, and how, for instance, the index of dispersion of the traffic process relates to the probabilities in the RHS of the above formula.

The event $u_{k-l}=0$ implies - at least - that no sources were active in the slot preceding slot $k-l$. This latter observation is sufficient to determine the future evolution of the process. One can show that, for 
the $G I-G-\infty$ model,

$$
\begin{aligned}
& E\left[z^{p_{k}+p_{k-1}+\ldots+p_{k-l}} \mid u_{k-l}=0\right]= \\
& \quad \exp \left\{\lambda^{*} \sum_{n=0}^{l} \operatorname{Pr}\left[\tau_{A}>n\right](l+1-n) z^{n}(z-1)\right\}
\end{aligned}
$$

Introducing residues in (9), we get the following expression

$$
\begin{aligned}
\operatorname{Pr}\left[u_{k+1}>\right. & m]=\sum_{l=0}^{+\infty} \operatorname{Pr}\left[u_{k-l}=0\right] \sum_{k=m+l+1}^{+\infty} \operatorname{Res}\left[z^{-(k+1)}\right. \\
& \left.\exp \left\{\lambda^{*} \sum_{n=0}^{l} \operatorname{Pr}\left[\tau_{A}>n\right](l+1-n) z^{n}(z-1)\right\}\right]_{z=0}
\end{aligned}
$$

For a system in equilibrium, $\operatorname{Pr}\left[u_{k-l}=0\right]$ is given by $1-\rho$, a wellknown result from queueing theory. Hereby, $\rho$ is the load of the system and equals $\lambda_{T}$. It remains to be determined if replacing residues around $z=0$ by residues around the other singularities (poles or branches) of the function involved, will lead to 'practical' results, or if an accurate numerical transform inversion is feasible.

\subsection{A second approach}

The queueing of discrete-time on-off sources was studied by a slot-toslot approach in e.g. [20] for a finite number of sources (with geometric off-times), and in e.g. [21] for a infinite number of sources. The model in the latter paper is more general than the model of Section 5.2, in that the number of new sources becoming active during a slot can have an arbitrary distribution. The special case of a Poisson distribution then leads to the $G I-G-\infty$ arrival process considered here. It is noteworthy that the Poisson distribution has a number of properties which simplify the analysis and results to some extent.

The analysis can proceed as follows. Consider the joint pgf of $u_{k}$, the number of cells in the system, and of $v_{i, k}$, the numbers of messages in the equivalent $G I-G-\infty$ model which still contain $i$ cells, i.e., which will still generate a single arrival per slot during the $i$ slots to come. One can then establish the following recurrence relation

$$
\begin{aligned}
& P_{k+1}\left(z, x_{1}, x_{2}, \ldots\right)=E\left[z^{u_{k+1}} x_{1}^{v_{1, k+1}} x_{2}^{v_{2, k+1}} \ldots\right] \\
& =z^{-1} \exp \left\{\lambda^{*} \sum_{k=1}^{+\infty} a(k)\left(x_{k}-1\right)\right\}
\end{aligned}
$$




$$
\cdot\left(P_{k}\left(z, z, z x_{1}, z x_{2}, \ldots\right)+(z-1) P_{k}\left(0, z, z x_{1}, z x_{2}, \ldots\right)\right)
$$

The pgf $P_{k}\left(0, x_{1}, x_{2}, \ldots\right)$ can easily be obtained by observing that the queue being empty at the beginning of a slot implies no messages arrived during the previous slot. This implies that the only messages in the GI$G-\infty$ queue are new messages. This straightforwardly leads to

$$
P_{k}\left(0, x_{1}, x_{2}, \ldots\right)=\operatorname{Pr}\left[u_{k}=0\right] \exp \left\{\lambda^{*} \sum_{k=1}^{+\infty} a(k)\left(x_{k}-1\right)\right\}
$$

We will not go further into the details of the analysis here, but it is possible to derive from this an expression for the mean buffer contents in regime. It is

$$
\lim _{k \rightarrow \infty} E\left[u_{k}\right]=\rho+\frac{\rho^{2}}{2(1-\rho)}\left(E\left[\tau_{A}\right]+\frac{\sigma_{A}^{2}}{E\left[\tau_{A}\right]}\right)
$$

This expression can also be found from that in [20] by a limiting procedure, or from that in [21] by assuming a Poisson arrival process for new messages. The formula contains the variance of the durations of the on-periods and becomes infinite for heavy-tailed on-time distributions having infinite variance.

We believe - but couldn't prove yet - that, in general,

$$
\operatorname{Pr}\left[\tau_{A}=m\right] \sim m^{-q} \Rightarrow \operatorname{Pr}[u>m] \sim m^{-(q-2)}
$$

while

$$
\operatorname{Pr}\left[\tau_{A}=m\right] \sim z_{0}^{-m} \Rightarrow \operatorname{Pr}[u>m] \sim z_{0}^{*-m}
$$

Similar observations have been made for fluid-flow models [2]. Of course, in order for a result like (12) or (13) to be of 'practical' value, one should also be able to derive the constant of proportionality, i.e., the 'intercept' of the curve $m^{-(q-2)}$. In e.g. [21] it was assumed that the dominating singularity of pgf of the system contents is an isolated pole, somewhere in the interval $(1,+\infty)$ of the real line, what then leads to geometric tail decay. However, this assumption is no longer valid when heavy-tailed on-time distributions are involved, since the corresponding pgf's have a branchpoint at $z=1$. It remains to be studied how this approach has to be modified to deal with that case. 
Both approaches presented here, the Benes approach and the slotto-slot approach, are naturally related, since both pertain to the same model. The connection becomes apparent when one recurses the relation (11) (infinitely) many times, and sets all $x_{i}$ equal to one. This yields

$$
\begin{aligned}
E\left[z^{u_{k+1}}\right]=\lim _{M \rightarrow \infty}\left[\sum_{l=0}^{M} \operatorname{Pr}\left[u_{k-l}=0\right] z^{-(l+1)}\right. \\
\cdot \exp \left\{\lambda^{*} \sum_{n=0}^{l} \operatorname{Pr}\left[\tau_{A}>n\right](l+1-n) z^{n}(z-1)\right\} \\
+z^{-(M+1)} \exp \left\{\lambda^{*} \sum_{n=0}^{M-1} \operatorname{Pr}\left[\tau_{A}>n\right](M-n) z^{n}(z-1)\right\} \\
\left.\cdot P_{k-M}\left(z, z, z^{2}, z^{3}, \ldots, z^{M}, z^{M+1}, z^{M+1}, \ldots\right)\right]
\end{aligned}
$$

Comparing this with equation (10), one easily recognizes the terms they have in common. However, establishing how they converge exactly, still requires further study.

\section{Conclusions}

A number of results were presented concerning traffic characteristics and queueing behavior of discrete-time on-off sources. At various instances, the distinction between short-range and long-range dependent traffic was touched upon. Some key issues remain unsolved, and, as such, create challenging areas for future research.

In its strictest sense, long-range dependence is present when e.g. the on-period distribution of the sources has an infinite variance. This leads to an infinite 'DC-component' in the power spectral density, a system contents having infinite mean, etcetera. However, to the authors' opinion, the distinction between geometric tail decay and hyperbolic tail decay is as important as that between short- and long-range dependence. A relation like (12) shows that the system contents can still have a slowly decaying tail, even when the distributions of the sources have a finite variance and when, as such, the system contents has a finite mean. Also for this type of 'short-range dependent' traffic, a tremendous amount of buffering might be needed in order to avoid cell loss, or, in other words, to allow for feasible statistical multiplexing. 


\section{Appendix A}

In this appendix, an expressions is derived for

$$
S(f)=\sum_{m=-\infty}^{+\infty} C(m) e^{j 2 \pi m f}
$$

whereby $C(m)$ was defined as $E\left[\left(q_{0}-\lambda\right)\left(q_{m}-\lambda\right)\right]$. Since there is at most one arrival per slot, one has $E\left[q_{0} q_{m}\right]=\operatorname{Pr}\left[\left(q_{0}=1\right) \&\left(q_{m}=1\right)\right]$. This can be further expressed as

$$
\sum_{k=0}^{+\infty} \operatorname{Pr}\left[q_{m}=1 \mid\left(q_{0}=1\right) \&\left(\tau_{A}^{*}=k\right)\right] \operatorname{Pr}\left[\left(q_{0}=1\right) \&\left(\tau_{A}^{*}=k\right)\right]
$$

whereby $\tau^{*}$ denotes the number of remaining slots of the on- or offperiod in which slot 0 falls (not counting slot 0 itself). The factor $\operatorname{Pr}\left[\left(q_{0}=1\right) \&\left(\tau^{*}=k\right)\right]=\operatorname{Pr}\left[\tau^{*}=k \mid q_{0}=1\right] \operatorname{Pr}\left[q_{0}=1\right]$ can be expressed as $a^{*}(k) \lambda$. Recall - see section 2 - that the pgf associated with the distribution $a^{*}(k)$ is $A^{*}(z)=[A(z)-1] /\left[A^{\prime}(1)(z-1)\right]$.

The probability $\operatorname{Pr}\left[q_{m}=1 \mid\left(q_{0}=1\right) \&\left(\tau_{A}^{*}=k\right)\right]$ is given by

$$
\operatorname{Pr}\left[q_{m}=1 \mid\left(q_{0}=1\right) \&\left(\tau_{A}^{*}=k\right)\right]= \begin{cases}1 & : m \leq k \\ \operatorname{Pr}\left[q_{m-k}=1 \mid B_{1}\right]: & m>k\end{cases}
$$

whereby $B_{1}$ was used to denote the event that slot 1 , i.e., the slot just after slot 0 , is the first slot of an off-period. By considering all possible values for the durations of that off-period, with proper weights, one finds

$$
\begin{aligned}
& \operatorname{Pr}\left[q_{m}=1 \mid B_{1}\right]= \\
& \sum_{k=1}^{+\infty} b(k)\left\{I(m \leq k) \cdot 0+I(m>k) \cdot \operatorname{Pr}\left[q_{m-k}=1 \mid A_{1}\right]\right\}
\end{aligned}
$$

Similar as above, $A_{1}$ denotes the event that slot 1 is the first slot of an on-period. $I($.$) denotes the indicator function. The probability \operatorname{Pr}\left[q_{m}=\right.$ $\left.1 \mid A_{1}\right]$ can, likewise, be expressed as

$$
\begin{aligned}
& \operatorname{Pr}\left[q_{m}=1 \mid A_{1}\right]= \\
& \sum_{k=1}^{+\infty} a(k)\left\{I(m \leq k) \cdot 1+I(m>k) \cdot \operatorname{Pr}\left[q_{m-k}=1 \mid B_{1}\right]\right\}
\end{aligned}
$$


Introducing z-transforms in equations (16) and (17), and performing some algebra, one can show that

$$
X_{B}(z)=\sum_{m=1}^{+\infty} \operatorname{Pr}\left[q_{m}=1 \mid B_{1}\right] z^{m}=z \frac{A(z)-1}{z-1} \cdot \frac{B(z)}{1-A(z) B(z)}
$$

and

$$
X_{A}(z)=\sum_{m=1}^{+\infty} \operatorname{Pr}\left[q_{m}=1 \mid A_{1}\right] z^{m}=z \frac{A(z)-1}{z-1} \cdot \frac{1}{1-A(z) B(z)}
$$

Returning to equations (14) and (15), one obtains

$$
\sum_{m=1}^{+\infty} E\left[q_{0} q_{m}\right] z^{m}=\lambda\left(z \frac{A^{*}(z)-1}{z-1}+A^{*}(z) X_{B}(z)\right)
$$

and, after some further manipulation,

$$
\sum_{m=1}^{+\infty} C(m) z^{m}=\sigma^{2} Q(z)=\sigma^{2} z \frac{P(z)-1}{z-1}
$$

whereby $\sigma^{2}=\operatorname{Var}\left[q_{k}\right]=\lambda(1-\lambda)$ and

$$
P(z)=\frac{A(z)-1}{A^{\prime}(1)(z-1)} \cdot \frac{B(z)-1}{B^{\prime}(1)(z-1)} \cdot \frac{\left[A^{\prime}(1)+B^{\prime}(1)\right](z-1)}{A(z) B(z)-1}
$$

Finally, one obtains equation (1)

$$
S(f)=\sigma^{2}\left(1+Q\left(e^{j 2 \pi f}\right)+Q\left(e^{-j 2 \pi f}\right)\right)
$$

\section{Appendix B}

In this appendix, an expression is derived for

$$
J(z, t)=\sum_{m=1}^{+\infty} t^{m} E\left[z^{q_{1}+\ldots+q_{m}}\right]
$$

The derivations are quite similar to those in appendix A. Starting point is the expression

$$
J(z, t)=\frac{A^{\prime}(1)}{A^{\prime}(1)+B^{\prime}(1)} J_{A}(z, t)+\frac{B^{\prime}(1)}{A^{\prime}(1)+B^{\prime}(1)} J_{B}(z, t)
$$


whereby

$$
J_{A}(z, t)=\sum_{m=1}^{+\infty} t^{m}\left(\sum_{k=0}^{+\infty} a^{*}(k) E\left[z^{q_{1}+\ldots+q_{m}} \mid\left(q_{0}=1\right) \&\left(\tau_{A}^{*}=k\right)\right]\right)
$$

and

$$
J_{B}(z, t)=\sum_{m=1}^{+\infty} t^{m}\left(\sum_{k=0}^{+\infty} b^{*}(k) E\left[z^{q_{1}+\ldots+q_{m}} \mid\left(q_{0}=0\right) \&\left(\tau_{B}^{*}=k\right)\right]\right)
$$

It is easily shown that

$$
E\left[z^{q_{1}+\ldots+q_{m}} \mid\left(q_{0}=1\right) \&\left(\tau_{A}^{*}=k\right)\right]= \begin{cases}z^{m} & : m \leq k \\ z^{k} E\left[z^{q_{1}+\ldots+q_{m-k}} \mid B_{1}\right]: & m>k\end{cases}
$$

and

$$
E\left[z^{q_{1}+\ldots+q_{m}} \mid\left(q_{0}=0\right) \&\left(\tau_{B}^{*}=k\right)\right]= \begin{cases}1^{m} & : m \leq k \\ 1^{k} E\left[z^{q_{1}+\ldots+q_{m-k}} \mid A_{1}\right] & : m>k\end{cases}
$$

As in the previous appendix, $B_{1}$ and $A_{1}$ denote the events that slot 1 is the first slot of an off- or an on-period respectively.

One has

$$
\begin{aligned}
& E\left[z^{q_{1}+\ldots+q_{m}} \mid B_{1}\right]= \\
& \sum_{k=1}^{+\infty} b(k)\left\{I(m \leq k) \cdot 1^{m}+I(m>k) \cdot 1^{k} E\left[z^{q_{1}+\ldots+q_{m-k}} \mid A_{1}\right]\right\}
\end{aligned}
$$

and

$$
\begin{aligned}
& E\left[z^{q_{1}+\ldots+q_{m}} \mid A_{1}\right]= \\
& \sum_{k=1}^{+\infty} a(k)\left\{I(m \leq k) \cdot z^{m}+I(m>k) \cdot z^{k} E\left[z^{q_{1}+\ldots+q_{m-k}} \mid B_{1}\right]\right\}
\end{aligned}
$$

Introducing a $z$-transform (in variable $t$ ) in the above equations and performing some straightforward algebra, one obtains

$$
\begin{aligned}
K_{A}(z, t) & =\sum_{m=1}^{+\infty} t^{m} E\left[z^{q_{1}+\ldots+q_{m}} \mid A_{1}\right] \\
& =\frac{1}{1-A(z t) B(t)}\left(z t \frac{A(z t)-1}{z t-1}+A(z t) t \frac{B(t)-1}{t-1}\right)
\end{aligned}
$$


and

$$
\begin{aligned}
K_{B}(z, t) & =\sum_{m=1}^{+\infty} t^{m} E\left[z^{q_{1}+\ldots+q_{m}} \mid B_{1}\right] \\
& =\frac{1}{1-A(z t) B(t)}\left(t \frac{B(t)-1}{t-1}+B(t) z t \frac{A(z t)-1}{z t-1}\right)
\end{aligned}
$$

Further,

$$
\begin{aligned}
J_{A}(z, t) & =z t \frac{A^{*}(z t)-1}{z t-1}+A^{*}(z t) K_{B}(z, t) \\
& =\frac{z t}{1-z t}+\frac{t A^{*}(z t)(B(t)-1)(z-1)}{(z t-1)(t-1)(1-A(z t) B(t))}
\end{aligned}
$$

and

$$
\begin{aligned}
J_{B}(z, t) & =t \frac{B^{*}(t)-1}{t-1}+B^{*}(t) K_{A}(z, t) \\
& =\frac{t}{1-t}-\frac{t B^{*}(t)(A(z t)-1)(z-1)}{(z t-1)(t-1)(1-A(z t) B(t))}
\end{aligned}
$$

Inserting these expressions in equation (18), one finally obtains

$$
\begin{aligned}
& J(z, t)=\frac{1}{A^{\prime}(1)+B^{\prime}(1)}\left(\frac{A^{\prime}(1) z t}{1-z t}+\frac{B^{\prime}(1) t}{1-t}\right. \\
& \left.-\frac{A^{\prime}(1) B^{\prime}(1) t^{2}(z-1)^{2} A^{*}(z t) B^{*}(t)}{(1-z t)(1-t)(1-A(z t) B(t))}\right)
\end{aligned}
$$

\section{References}

[1] J. Beran, R. Sherman, M. Taqqu and W. Willinger, "Long-range dependence in variable-bit-rate video traffic", IEEE Transactions on Communications 43 (1995) 1566-1579.

[2] O.J. Boxma, "Fluid queues and regular variation", Proceedings of PERFORMANCE'96 (Lausanne, October 1996) Performance Evaluation 27\&28 (1996) 699-712.

[3] H. Bruneel and B.G. Kim, Discrete-time models for communication systems including ATM, Kluwer Academic Publishers (1993). 
[4] G.L. Choudhury en W. Whitt, "Computing distributions and moments in polling models by numerical transform inversion", Performance Evaluation 25 (1996) 267-292.

[5] R. Grünenfelder and S. Robert, "Which arrival law parameters are decisive for queueing system performance", Proceedings ITC-14 (Antibes Juan-les-Pins, June 1994) 377-386.

[6] R. Gusella, "Characterizing the variability of arrival processes with indexes of dispersion", IEEE Journal on Selected Areas in Communications 9 (1991) 203-211.

[7] J.J. Hunter, Mathematical techniques of applied probability, Volume 2, discrete time models: techniques and applications, Academic Press Inc. (1983).

[8] P. Jelenković and A. Lazar, "Multiplexing on-off sources with subexponential on periods: part I", Proceedings INFOCOM'97 (Kobe, April 1997) 187-195.

[9] P. Jelenković and A. Lazar, "Multiplexing on-off sources with subexponential on periods: part II", Proceedings ITC15 (Washington D.C., June 1997) 965-974.

[10] W.E. Leland, M.S. Taqqu, W. Willinger and D.V. Wilson, "On the self-similar nature of ethernet-traffic (extended version)", IEEE/ACM Transactions on Networking 2 (1994) 1-15.

[11] S.-Q. Li and C.-L. Hwang, "Queue response to input correlation functions: discrete spectral analysis", IEEE/ACM Transactions on Networking 1 (1993) 522-533.

[12] I. Norros, J.W. Roberts, A. Simonian and J.T. Virtamo, "The superposition of variable bit rate sources in an ATM multiplexer", IEEE Journal on Selected Areas in Communications 9 (1991) 378387.

[13] I. Norros, "A storage model with self-similar input", QUESTA 16 (1994) 387-396.

[14] I. Norros, "On the use of fractional Brownian motion in the theory of connectionless networks", IEEE Journal on Selected Areas in Communications 13 (1995) 953-962.

[15] M. Parulekar and A. Makowski, "M/G/ $\infty$ input processes: a versatile class of models for network traffic", Proceedings INFOCOM'97 (Kobe, April 1997) 419-426. 
[16] V. Paxson and S. Floyd, "Wide area traffic: the failure of Poisson modeling", IEEE/ACM Transactions on Networking 3 (1995) 226244.

[17] W. Press, S. Teukoslky, W. Vetterling and B. Flannery, Numerical Recipes in C (second edition), Cambridge University Press (1994).

[18] J. Roberts, U. Mocci and J. Virtamo (editors), Broadband network teletraffic, COST 242 Final Report, Springer-Verlag (1996).

[19] K. Tsoukatos and A. Makowski, "Heavy traffic analysis for a multiplexer driven by M/GI/ $\infty$ input processes", Proceedings ITC15 (Washington D.C., June 1997) 497-506.

[20] S. Wittevrongel and H. Bruneel, "Effect of the on-period distribution on the performance of an ATM multiplexer fed by on/off sources : an analytical study", Proceedings of the 6th IFIP WG 6.3 Conference on Performance of Computer Networks, PCN '95 (Istanbul, October 1995) 33-47.

[21] S. Wittevrongel and H. Bruneel, "Queue length and delay for statistical multiplexers with variable-length messages", Proceedings of GLOBECOM'94 (San Francisco, November 1994) 1080-1084. 